\title{
Research on LTE Random Access Process
}

\author{
QIU feng
}

Chongqing College of Electronic Engineering, Chongqing, China, 401331

63117131@qq.com

Keywords: LTE, Random Access Process, Communication Standards

\begin{abstract}
This paper first summarizes the development and evolution of LTE system and cluster communication system, analyzes the random access technology in LTE system, summarizes the access flow of cluster system, compares the random access and random access of LTE In order to access the LTE system, it is necessary for the cluster users to access the same as the ordinary users, according to the current LTE access process. Because the cluster users are grouped, they will occupy the access channel resources in a short time when they access the LTE network, and may cause the access collision and delay, which affects the real-time performance of the communication of the cluster users. Therefore, the traditional LTE random access mechanism can not meet the cluster user's access requirements.
\end{abstract}

\section{Introduction}

With the rapid development of social economy and the continuous progress of communication technology, the cost of the cluster system construction project is gradually reduced. More and more individuals and enterprises and institutions can start to bear the cost of using the cluster communication system, and can realize the cluster System in real-time communication, security and other aspects of the great advantages, so small-scale private cluster communications system began to flourish. With the rapid growth of demand for broadband communication system, the government has taken the lead in enterprise, and the cluster communication system has been gradually trying to carry on the public network communication system. Typical examples include i DEN, which is built by Shanghai and Shenzhen joint ventures. System, the Beijing Municipal Government builds the TETRA system. The emergence of the broadband cluster system on the public network communication system largely alleviated the burden of individuals or enterprises to build their own private small cluster system, and then by the use of more units sought after, with more rapid development. With the birth of the public network cluster system, but also with the public network cluster operators, public network cluster system, the number of users continues to increase, to the operator's operating capacity and public network cluster system networking and communication capabilities higher requirement. In the first generation of simulated cluster communications boom, China has not yet started cluster communication technology research, this situation to the second generation of digital trunking communication development has not changed much at the beginning, until the emergence of broadband digital trunking system, China Of the cluster communication technology began to develop rapidly, more well-known broadband cluster communications systems, including ZTE Corporation developed Go Ta digital trunking communication system and Huawei Communication Technology Co., Ltd. developed GT8000 digital trunking communication system.

LTE cluster communication is different from ordinary LTE communication, its service real-time business more for access management, resource allocation speed and other requirements compared to ordinary LTE communication more stringent. Therefore, to build LTE cluster communication system, it is necessary on the basis of LTE, according to the cluster system business needs, its access control, resource allocation, switching control and other aspects of the performance of each side to further enhance. With Chinese entry into the LTE era, broadband R \& D system has also entered a new stage. In order to seize the development of LTE cluster system, in the cluster standards and intellectual property rights to regain the initiative, the state in the field of cluster communications into a considerable scientific research, and joint universities, well-known communications companies to develop and promote LTE cluster communication standards With the 
system, has achieved initial results. At present, the more well-known research and development of LTE cluster standards and systems, including Chinese Putian proposed Wi TRA, Huawei's e LTE and so on. However, because LTE cluster communication protocol and system in China and around the world is still in the research stage, so there is no unified LTE cluster system communication standards and protocols. The research of this paper is mostly based on the current research in the cluster system and the existing LTE communication network protocol and started.

\section{LTE random access technology analysis}

In LTE, the random access procedure provides six functions: 1) establishes a wireless link at initial access. 2) Reestablish the radio link after the link establishment fails. 3) After the switch, the new terminal establishes uplink synchronization. 4) When the terminal reaches RRC_CONNECTED and the uplink is not synchronized, uplink data is established when uplink data or downlink data arrives. 5) positioning by measuring the positioning method of the uplink. 6) When the PUCCH is not equipped with a dedicated scheduling response resource, it is used as a scheduling response.

According to whether the UE is in the uplink synchronization state when the access is initiated, the random access procedure can be divided into asynchronous random access procedure and synchronous random process. The asynchronous random access procedure means that the UE does not acquire uplink synchronization or has lost the random access procedure in the uplink synchronization state. The synchronous random access procedure means that when the UE has obtained the uplink synchronization, when the uplink resource is requested or the handover is performed or otherwise Of the random access process. Depending on the scene of the random access scenario, random access can be divided into contention-based random access and non-contention based random access. The noncompetitive random access procedure is only applicable to a specific trigger scenario, such as handover and downlink data has been reached and the UE has not yet established an uplink synchronization with the e Node B, and the rest can use a contention based random access procedure The

The collision is mainly due to the fact that more than one UE chooses to send the same preamble on the same time-frequency resource, at which point the base station replies to the same random access response to all users who send the preamble, including the same temporary C-RNTI The It is because of the existence of the collision, HARQ was not used for random access response. After the collision of the UE in accordance with the instructions in the RAR on the same upstream time-frequency resources send Message3, when the collision will occur. This time the collision may cause the collided UE to not be decoded, further causing the UE to initiate a random access when the HARQ reaches the maximum number of retransmissions. However, if one or several UEs are successfully decoded, the collision is still not resolved for other UEs. So to continue the competitive solution is a very necessary process, which is achieved through Message4. Message4 contains the UEID carried in Message3, which satisfies the successful UE, and supports HARQ. When a collision occurs and e Node B successfully decodes Message3, the UE detects its own UEID, sends HARQ feedback to the e Node B, and other UEs that fail to resolve the session will quickly exit the current random access procedure. The backoff time is reset and the new random access is restarted.

\section{LTE cluster system users fast random access method}

There is a lot of demand for LTE cluster systems in the industry, but because LTE is a new public network system, there is very little reference to LTE cluster system and no description of cluster user access in LTE has been found. Through the analysis of the second chapter, we can see how the rapid access of TD-LTE to cluster system is of great significance. If the cluster user is connected to the LTE system as a normal user, a large number of cluster users will access in a short period of time. Each user can randomly select the preamble to send the access request. There will be collisions between users in the cluster user group, and the sudden increase in cluster users will improve the likelihood of collision when they are ready for access. In this paper, we use the group of cluster users to design a fast random access method for LTE cluster system users. 
When using this method for random access, the cluster first selects a cluster agent access user, which represents the entire cluster user group access to the LTE system, the remaining users for the cluster of ordinary access users, monitoring cluster dedicated channel waiting to receive the cluster agent accesses the access information sent by the user. After the cluster agent user starts to interact with the base station according to the normal random access procedure: firstly sends the agent random access request and receives the RAR message returned by the base station; then sends the agent dispatch transmission information and receives the competition resolution success information returned by the base station Connection establishment state; Finally, in the cluster dedicated channel broadcast cluster access to ordinary users access information, the cluster of ordinary access to the user after receiving the information into the connection establishment state, the random access process ends. Among them, the cluster-specific channel is a channel designed by the author according to the characteristics of the cluster communication in combination with the traditional cluster system mode. The channel can use the specific frequency band inside the cluster to realize the intercommunication between different UEs under the same base station. Channel broadcast information, the cluster of ordinary access users by listening to the channel to receive access information. The following is a detailed description of each step of the method.

According to the boot time has to select the cluster agent access users. After the user in the cluster group is powered on, it first listens on the cluster dedicated channel. If the listening time of the user has been monitored by the user at the beginning of the T1 time, the cluster agent accesses the user to send the information of the success of the access. If the access success message is heard during the T1 time, the cluster agent into the user on behalf of all users' access to success, receive relevant information directly from the access into the connection state. The above two cases show that the user is a cluster of ordinary access users, just receive the access success information, send an ACK message to enter the connection establishment state. If the T1 time does not listen to any message, then think that he is the first boot user, a cluster agent access to the user, responsible for the agent cluster group of all users' access. And then broadcasts the random access start message on the cluster dedicated channel with $\mathrm{T} 1$ as a cycle so that other users can listen. If two users at the same time boot, and that they are a cluster agent access users, then the base station to determine which of the two users who is a cluster agent access users.

Since the proxy random access request needs to include each user's preamble, the cluster agent user needs to obtain a preamble for each user. The cluster agent access user first receives the configuration information transmitted by the base station on the broadcast channel and obtains the packet of the UE preamble. And then select $\mathrm{N}$ different preambles to the base station in the available preamble sequences according to the total number $\mathrm{N}$ of the users to be sent in the cluster group, so that the same preamble can be avoided as a result of the selection of the same preamble into the collision. When $\mathrm{N}$ is greater than the number of preamble available, the cluster agent access user groups the cluster access users according to the device number, and each time a group of ordinary access users of the cluster accesses random access until all the cluster access users are proxied Access is successful. After the Preamble is selected, the proxy cluster access request is sent to the base station and is waiting to receive the RAR. By the steps of the fast random access method of the LTE cluster system, it is known that when the access request is transmitted, the access agent is selected by the cluster agent to select the $\mathrm{N}$ available preamble according to the number of users currently being accessed, Node B. The number of available preamble for each access slot is limited. Suppose that there are 64 available preambles in a time slot. There are 32 cluster users in the current time slot. Ordinary users randomly select an initiator in 64 preamble and there is a $50 \%$ chance of colliding with the cluster user. It can be found that when a cluster user accesses, the preamble selected by the normal user has a high probability that it will conflict with the preamble of the cluster user. And the base station side will give priority to determine the success of cluster users' access, so the collision of ordinary users in the competition to resolve the probability of failure is very large. The user who fails to access the connection will start again after the random access request, and LTE back-off mode is not back, that is, the failure of the user will be in the next access slot again to apply for access and it is possible again so large-scale collision, resulting in another 
access failure. Therefore, it can be concluded that in the system using the fast random access method of the LTE cluster system, the system fairness is poor and the primary cluster access may have the effect of continuous access to the ordinary users.

\section{Conclusion}

The fast random access method of the LTE cluster system user is a fast access method which uses a user instead of a group of users to access the other users in the group. This method can access several cluster users at the same time in the access process, greatly reducing the access time of the cluster users, avoiding the failure of the networking caused by the unsuccessful access of individual cluster users, or the communication limit. The validity of this method is verified by simulation. But the simulation also exposed the LTE cluster system users fast random access method is a sacrifice of the average user access efficiency of the unfair access method.

\section{References}

[1] Chi Haixiang. Implementation of cluster in LTE network [J]. Mobile communication. 2014 (20)

[2] Tang Hong, Li Min, Zhou Tuo. Random access algorithm based on priority partition of TD-LTE cluster communication system [J]. Journal of Chongqing University of Posts and Telecommunications (Natural Science Edition)

[3] Liao Shengbin, Zhu Xiaoliang. Backoff algorithm based on optimal window in IEEE 802.11 wireless local area network [J] .Computer Science. 2012 (01)

[4] Yang Bo, Liao Jianxin, Jiang Mingzhe. Design and implementation of cluster communication system based on public mobile communication network [J] .Technology \&

[5] Xue Liang, Qian Liang. Study on random access based on Bayesian criterion [J] .Technology and Communication Technology, 2005 (04) 